\title{
Testing the ecological validity of the Trier Social Stress Test: Association with real-life exam stress
}

\author{
Gina-Isabelle Henze ${ }^{a}$, Sandra Zänkert ${ }^{a}$, David F. Urschler ${ }^{\mathrm{a}}$, Tanja J. Hiltl ${ }^{\mathrm{a}}$, \\ Brigitte M. Kudielka ${ }^{a}$, Jens C. Pruessner ${ }^{b}$, Stefan Wüst ${ }^{a, *}$ \\ a Institute of Experimental Psychology, University of Regensburg, Universitätsstraße 31, 93053 Regensburg, Germany \\ ${ }^{\mathrm{b}}$ Department of Psychology, McGill University, 845 Rue Sherbrooke O, Montréal, QC H3A 0G4, Canada
}

Keywords:

Acute stress responses

HPA axis

Salivary cortisol

Positive/negative affect

Real-life stress

\begin{abstract}
A B S T R A C T
The Trier Social Stress Test (TSST) is the most widely used laboratory stress protocol in psychoneuroendocrinology. Despite its popularity, surprisingly few attempts have been made to explore the ecological validity of the TSST. In the present study, 31 young healthy subjects ( 24 females) were exposed to the TSST about 4 weeks before completing an oral exam on a separate day. Salivary cortisol levels increased significantly in response to both stimuli $($ TSST: $F(2.21,66.33)=5.73, p=0.004$; oral exam: $F(1.98,59.28)=4.38$, $\mathrm{p}=0.017$ ) with similar mean response curves and significant correlations between cortisol increases and areas under the response curves (increase: $\mathrm{r}=0.67$; AUC: $\mathrm{r}=0.56$; both $\mathrm{p} \leq 0.01$ ). Correspondingly, changes in positive and negative affect did also show significant correlations between conditions (increase: positive affect: $r=0.36$; negative affect: $r=0.50$; both: $\mathrm{p} \leq 0.05 ;$ AUC: positive affect: $\mathrm{r}=0.81$; negative affect: $\mathrm{r}=0.70$; both $\mathrm{p} \leq 0.01$ ) while mean time course dynamics were significantly different (positive affect: $\mathrm{F}(2.55,76.60)=10.15, \mathrm{p}=0.001$; negative affect: $\mathrm{F}(1.56,46.82)=23.32, \mathrm{p}=0.001)$, indicating that the oral exam had a more pronounced impact on affect than the TSST.

Our findings provide new evidence for the view that cortisol as well as subjective stress responses to the TSST are indeed significantly associated with acute stress responses in real life.
\end{abstract}

\section{Introduction}

Hypothalamus-pituitary-adrenal (HPA) axis responses to acute stress are considered an important biomarker of individual stress regulation, more so than baseline measures. Therefore, psychoneuroendocrine research on the link between stress and disease in humans requires standardized and valid protocols to induce psychological stress under controlled conditions. A protocol that has widely been used in healthy subjects as well as in various clinical populations for more than 20 years is the Trier Social Stress Test (TSST) (Kirschbaum et al., 1993, 1992; Kudielka et al., 2009). However, although stress exposure under controlled conditions has advantages in many respects, it lacks direct evidence of ecological

\footnotetext{
* Corresponding author.

E-mail addresses: Henze@psychologie.uni-regensburg.de (G.-I. Henze), Zaenkert@psychologie.uni-regensburg.de (S. Zänkert), Urschler@psychologie.uni-regensburg.de (D.F.Urschler), Hiltl@stud.uni-regensburg.de (T.J. Hiltl), Kudielka@psychologie.uni-regensburg.de (B.M. Kudielka), Pruessner@mcgill.ca (J.C. Pruessner),

Wuest@psychologie.uni-regensburg.de (S. Wüst).
}

validity (Dickerson and Kemeny, 2004). The usefulness of the TSST has been clearly demonstrated, e.g. in studies that found significant stress response differences between psychiatric patients and healthy controls (Burke et al., 2005) or in studies showing significant and plausible cross-correlations between psychological and endocrine stress responses (Schlotz et al., 2008). But to the best of our knowledge, very few attempts have been made to directly address the question, if stress responses to the TSST are related to acute stress responses in real life. An exception is a study by Wolfram et al. (2013) who investigated the association between cortisol responses to the TSST and responses to a graded demonstration lesson in a sample of 21 student teachers, but they failed to find a significant correlation. It is, however, possible that this study missed to capture the cortisol peak in the real-life stress condition because saliva sampling was not possible during the $45 \mathrm{~min}$ demonstration lesson.

To further explore the ecological validity of the TSST in the present study, we chose a brief oral exam as naturalistic stressor. The advantage of the current approach was the similarity to the TSST in duration $(10 \mathrm{~min})$ and content (speaking in front of an audience with the perceived risk of being negatively evalu- 
ated (Dickerson and Kemeny, 2004; Kudielka and Wüst, 2010)). We hypothesized that we would observe a significant association between the oral exam and the TSST with respect to cortisol and subjective stress responses.

\section{Materials and methods}

In a sample of 31 apparently healthy students of the University of Regensburg (24 women, 7 men; age: $19-32$ years, $M=22.48$, $S D=4.32$ ) we assessed salivary cortisol and self-reported psychological stress responses to the TSST and to an oral pass-fail exam that was part of a university course for bachelor students in psychology. This test was the first oral exam in the study course. While the panel consisted of two examiners in the TSST, only the student and the lecturer, inquiring about main topics of the psychology course, were present in the room during the oral exam. Both stress exposures took place on weekdays between 1:00 and 5:00 pm and subjects were instructed to refrain from physical exercise and large meals at least $90 \mathrm{~min}$ before the onset of the assessment. The exposures followed identical protocols including repeated saliva sampling with cortisol salivettes (Sarstedt, Nümbrecht, Germany) and affect ratings using the Positive and Negative Affect Scales (PANAS; (Watson et al., 1988)). Participants were asked to collect samples and to rate their affect at minute $-30,-20,-10,0,+10$, $+20,+30$ and +40 relative to stress onset. During the brief stress exposures, no saliva samples or affect ratings have been collected. All participants provided their written informed consent and were paid $20 €$ or received participant hours for taking part in the study. On average $26.7( \pm 12.47)$ days after TSST exposure subjects completed the oral exam as part of their bachelor's degree. As assessed with an in house questionnaire, all subjects reported good health and no psychological treatment for the past 24 months prior to study entry or scored below the cut-off value $(<14)$ of the WHO-5 World Health Organization Well-Being Index (WHO-5; (Topp et al., 2015)). Thirty participants reported to be non-smokers and one participant reported to smoke less than five cigarettes per day. Fourteen of the 24 females reported to use oral contraceptives. It was made clear to the subjects that participating in this study would in no way affect the outcome of the oral exam, and that the examiner was not involved in the study.

\section{Results}

An ANOVA for repeated measures with salivary cortisol as dependent measure yielded a significant main effect time for the $\operatorname{TSST}\left(F(2.21,66.33)=5.73, p=0.004, \eta^{2}=0.160\right)$ and the oral exam $\left(\mathrm{F}(1.98,59.28)=4.38, \mathrm{p}=0.017, \eta^{2}=0.127\right)$ (Fig. $\left.1 \mathrm{~A}\right)$. We applied Greenhouse-Geisser corrections because the sphericity assumptions were violated. While mean pre-stress levels in both conditions were virtually identical, cortisol responses to the TSST were marginally higher than responses to the oral exam (main effect condition: $\mathrm{F}(1.0,29.0)=3.56, \mathrm{p}=0.069, \eta^{2}=0.109$ ). This trend effect can be explained by higher TSST- compared to exam-responses in males as documented by a significant interaction effect of time $x$ condition $x \operatorname{sex}\left(\mathrm{F}(2.88,83.47)=3.46, \mathrm{p}=0.021, \eta^{2}=0.107\right)$. Responder rates according to the recently suggested criterion by Miller et al. (2013) were similar in the two stress exposures, with 22 subjects showing a cortisol response to the TSST and 18 subjects responding to the oral exam. We then used Pearson correlations to investigate the association of TSST cortisol responses and cortisol responses to our naturalistic stressor. We found that both, cortisol increases (i.e. individual peak levels minus individual pre-stress levels) as well as areas under the response curves $(A U C)$ as measure of total cortisol output were significantly correlated between TSST and exam con- dition (increase: $r=0.67, \eta^{2}=0.212 ;$ AUC: $r=.56, \eta^{2}=0.314$; both $\mathrm{p} \leq 0.01$; Fig. $1 \mathrm{~B}$ and C).

Furthermore, changes in positive and negative affect in response to the TSST and the oral exam were also significantly associated (increase: positive affect: $r=0.36, \eta^{2}=0.13$; negative affect: $r=0.50$, $\eta^{2}=0.25$; both: $\mathrm{p} \leq 0.05$; AUC: positive affect: $\mathrm{r}=0.81, \eta^{2}=0.66$; negative affect: $r=0.70, \eta^{2}=0.49$; both $p \leq 0.01$ ). Nevertheless, and in contrast to cortisol responses, mean time course dynamics in positive and negative affect did significantly differ between conditions (Fig. 2). An ANOVA for repeated measures revealed a significant time $x$ condition interaction for positive affect $(\mathrm{F}(2.55$, $\left.76.60)=10.15, p=0.001, \eta^{2}=0.25\right)$ as well as for negative affect changes $\left(F(1.56,46.82)=23.32, p=0.001, \eta^{2}=0.44\right)$. Positive affect levels prior to the oral exam where lower than prior to the TSST. They increased substantially after the oral exam while they did not show a rise after the TSST. Consistently, negative affect ratings where rather high prior to the oral exam followed by a distinct decline after stress. In contrast, the negative affect peak levels in the TSST condition were observed immediately after stress exposure has ended.

\section{Discussion}

The aim of the present study was to explore the ecological validity of the TSST, by comparing it with a real-life and meaningful acute psychological stressor. In a sample of 31 young healthy subjects, we found that salivary cortisol as well as affect responses to the TSST and to an oral university exam were significantly associated. These findings support the view that TSST responses indeed are significantly associated with acute stress responses in real life. Mean salivary cortisol response curves where very similar in both conditions suggesting a similar initial intensity of the overall perceived stress resulting in a subsequent HPA axis activation including a release of cortisol from the adrenals. However, mean affect response curves did significantly differ between conditions. While subjects reported to be rather nervous, anxious or distressed prior to the oral exam, their positive affect levels increased and their negative affect levels decreased immediately after it, a pattern that can perhaps be interpreted as significant relief. This assumption appears plausible as the subjects were immediately informed about their performance and as all subjects passed the ungraded exam. Remarkably, mean affect ratings clearly differed between conditions already at our first measurement point $(-30 \mathrm{~min})$. These pre-stress differences could indicate that the upcoming exam was appraised as being more relevant than the participation in a psychological study resulting in a more distinct anticipation response prior to the oral exam. Moreover, affect ratings prior to the TSST exposure may indicate that the subjects had no clear expectations regarding the TSST and that they thus underrated the stress induced by this "artificial" scenario (although the subjects certainly received a detailed written information describing the TSST-procedure before they signed the consent form). Affect ratings after the TSST did not suggest that the subjects felt relieved, perhaps as a consequence of forced failures and perceived social-evaluative threat. Due to the partial mismatch between expectations prior to exposure and actual situational characteristics of the TSST (e.g. two examiners not giving any (non-)verbal supportive feedback etc.) subjects may also have perceived more uncontrollability during the TSST than in the oral exam. This pattern can perhaps be described as 'worse than anticipated'. Although endocrine and psychological stress responses theoretically represent indicators of the same construct, an apparent inconsistency between these domains is a well-known phenomenon that, at least in part, can be explained by different response dynamics (Andrews et al., 2013; Schlotz et al., 2008). 

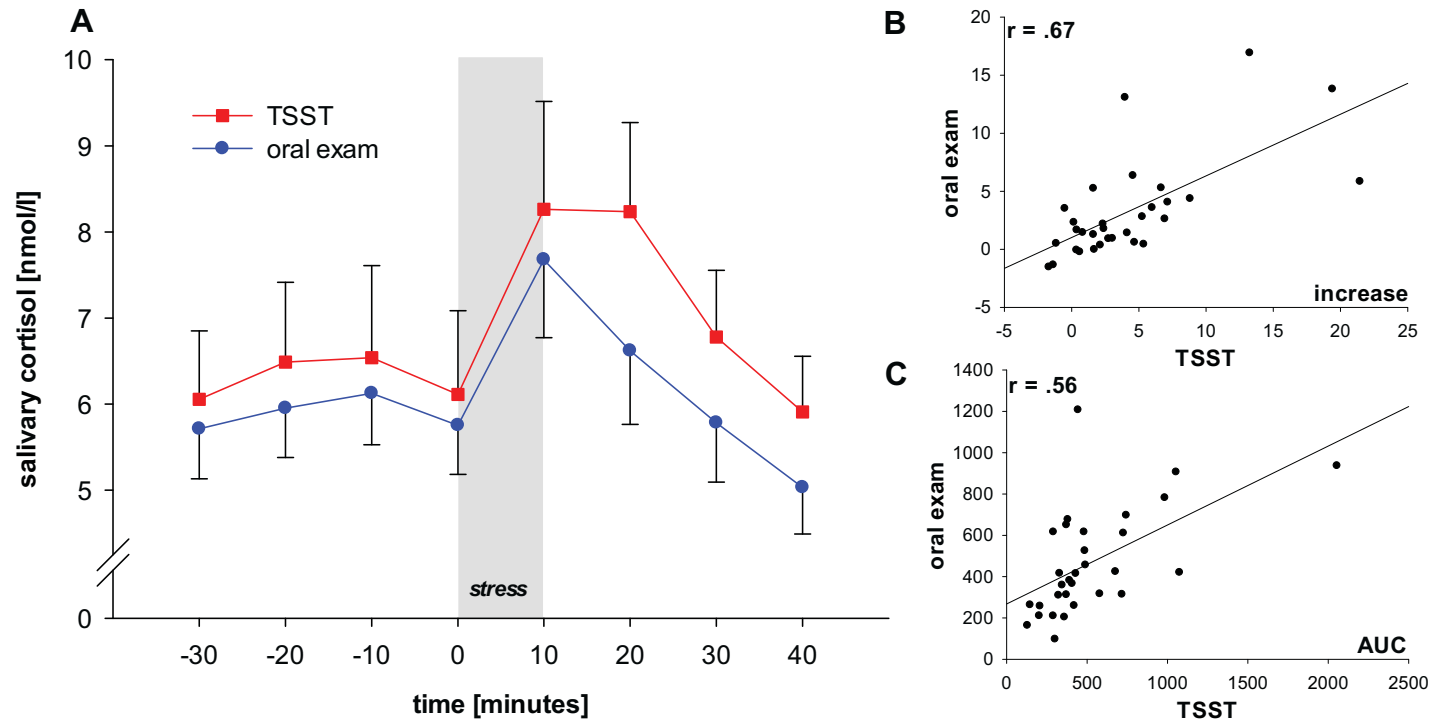

Fig. 1. (A) Mean salivary cortisol responses (nmo/l \pm SEM) to the TSST and the oral exam and correlations of cortisol (B) increases and (C) areas under the response curve (AUC) between the TSST and the oral exam.
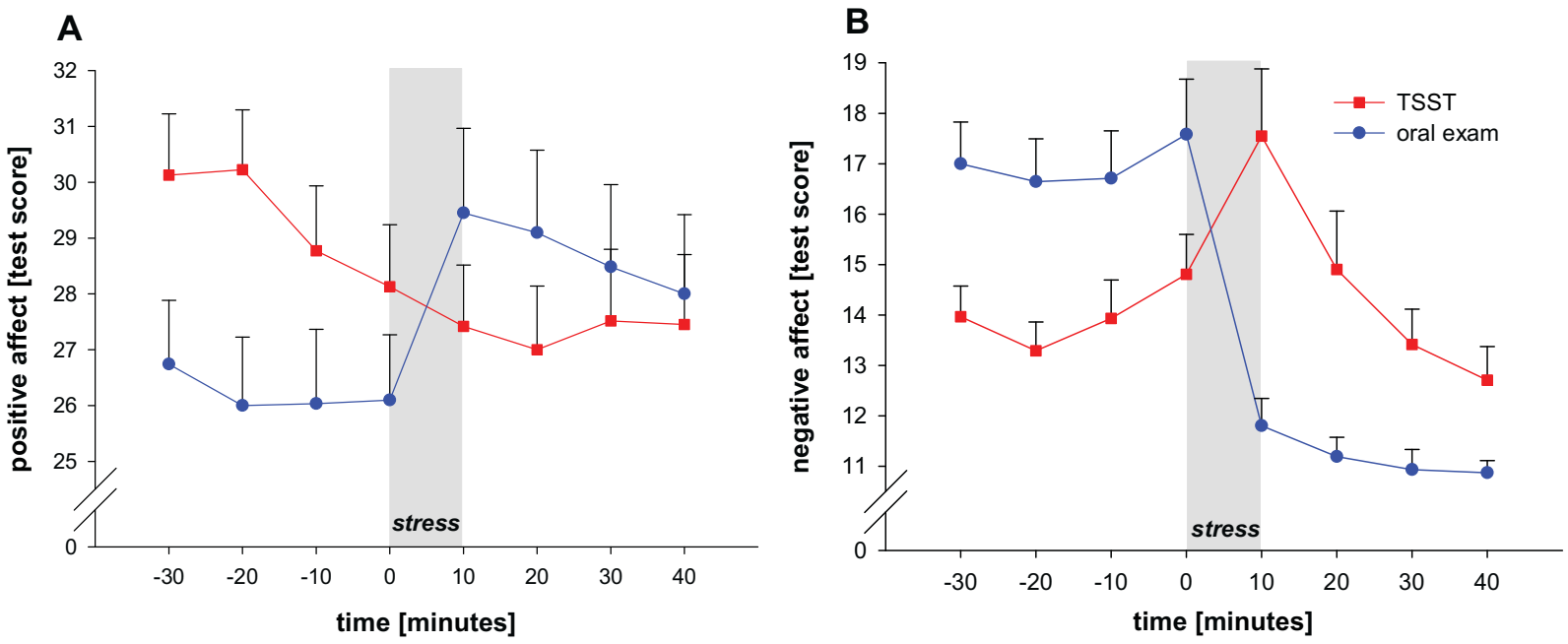

Fig. 2. Mean responses (score \pm SEM) of (A) positive and (B) negative affect (PANAS scores \pm SEM) to the TSST and the oral exam.

Our study has several limitations. First of all, the duration of our naturalistic stressor as well as its psychological elements (acute challenge, uncontrollability, pressure to perform, social-evaluative threat, fear of failure) were rather similar to those of the TSST. While this is not a limitation per se - in fact, we specifically searched for a naturalistic stressor with sufficient intensity that shows these characteristics - it limits the generalizability of our findings. Our findings do not demonstrate comprehensive ecological validity of the TSST for stress responses in general. Further obvious and interrelated limitations are the small sample size, particularly the small number of male subjects as well as the lack of control for oral contraceptive usage $(\mathrm{OC})$, of potential specific effects of different OCs (Pletzer and Kerschbaum, 2014) and of menstrual cycle phase including ovulation in female subjects. These shortcomings were caused by the fact that exams showing the above mentioned characteristics are rare at our institute and that only highly motivated students agree to participate in a study while taking an exam that is relevant for their bachelor's degree. However, in a supplementary analysis we did not find significant differences in mean cortisol responses to the TSST or the oral exam in females using versus not using oral contraceptives (all $\mathrm{p}>0.4$ for main effects $O C$ and time $x$
OC interactions). On a descriptive level, OC using females showed on average marginally lower cortisol levels prior to stress exposure while cortisol levels after stress exposure where almost identical.

In summary, the TSST has proven its usefulness in numerous studies. So far, however, our knowledge on the real-life validity of the TSST was scarce. In our view, the current study made an important contribution to the validation of this paradigm. Results of the present study suggest that salivary cortisol and affect responses to the TSST are significantly associated with corresponding responses to a brief acute stressor in real life.

\section{Contributors}

Gina-Isabelle Henze, Sandra Zänkert, David Urschler, Tanja J. Hiltl, Brigitte M. Kudielka, Jens C. Pruessner, and Stefan Wüst.

JCP, BMK and SW planned the study; GIH, SZ, DU and TJH prepared and performed the study; GIH and SW wrote the first draft of the manuscript; all authors contributed to the final version of the manuscript. 


\section{Conflict of interest}

All authors declare no conflict of interest related to this study.

\section{Role of the funding source}

This research did not receive any specific grant from funding agencies in the public, commercial, or not-for-profit sectors.

\section{Acknowledgements}

We thank Fabienne Ropeter, Petra Ebenschwanger and Corinna Stamminger for their help in running the experimental sessions and data entry.

This research did not receive any specific grant from funding agencies in the public, commercial, or not-for-profit sectors.

\section{References}

Andrews, J., Ali, N., Pruessner, J.C., 2013. Reflections on the interaction of psychogenic stress systems in humans: the stress coherence/compensation model. Psychoneuroendocrinology 38, 947-961.

Burke, H.M., Davis, M.C., Otte, C., Mohr, D.C., 2005. Depression and cortisol responses to psychological stress: a meta-analysis. Psychoneuroendocrinology 30, $846-856$

Dickerson, S.S., Kemeny, M.E., 2004. Acute stressors and cortisol responses: a theoretical integration and synthesis of laboratory research. Psychol. Bull. 130, 355-391.
Kirschbaum, C., Wüst, S., Faig, H.G., Hellhammer, D.H., 1992. Heritability of cortisol responses to human corticotropin-releasing hormone, ergometry, and psychological stress in humans. J. Clin. Endocrinol. Metab. 75, 1526-1530.

Kirschbaum, C., Pirke, K.M., Hellhammer, D.H., 1993. The 'Trier Social Stress Test' a tool for investigating psychobiological stress responses in a laboratory setting. Neuropsychobiology 28, 76-81.

Kudielka, B.M., Wüst, S., 2010. Human models in acute and chronic stress: assessing determinants of individual hypothalamus-pituitary-adrenal axis activity and reactivity. Stress 13,1-14.

Kudielka, B.M., Hellhammer, D.H., Wüst, S., 2009. Why do we respond so differently? Reviewing determinants of human salivary cortisol responses to challenge. Psychoneuroendocrinology 34, 2-18.

Miller, R., Plessow, F., Kirschbaum, C., Stalder, T., 2013. Classification criteria for distinguishing cortisol responders from nonresponders to psychosocial stress: evaluation of salivary cortisol pulse detection in panel designs. Psychosom. Med. 75, 832-840.

Pletzer, B.A., Kerschbaum, H.H., 2014. 50 years of hormonal contraception-time to find out, what it does to our brain. Front. Neurosci. 8, 256.

Schlotz, W. Kumsta, R., Layes, I., Entringer, S., Jones, A., Wüst, S., 2008. Covariance between psychological and endocrine responses to pharmacological challenge and psychosocial stress: a question of timing. Psychosom. Med. 70, 787-796.

Topp, C.W., Ostergaard, S.D., Sondergaard, S., Bech, P., 2015. The WHO-5 Well-Being Index: a systematic review of the literature. Psychother. Psychosom. 84, 167-176.

Watson, D., Clark, L.A., Tellegen, A., 1988. Development and validation of brief measures of positive and negative affect: the PANAS scales. J. Pers. Soc. Psychol. 54, 1063-1070.

Wolfram, M., Bellingrath, S., Feuerhahn, N., Kudielka, B.M., 2013. Cortisol responses to naturalistic and laboratory stress in student teachers: comparison with a non-stress control day. Stress Health 29, 143-149. 